Document downloaded from:

http://hdl.handle.net/10251/143324

This paper must be cited as:

Moreira, MC.; Pastor, R.; Costa, A.; Miralles Insa, CJ. (06-2). The multi-objective assembly line worker integration and balancing problem of type-2. Computers \& Operations Research. 82:114-125. https://doi.org/10.1016/j.cor.2017.01.003

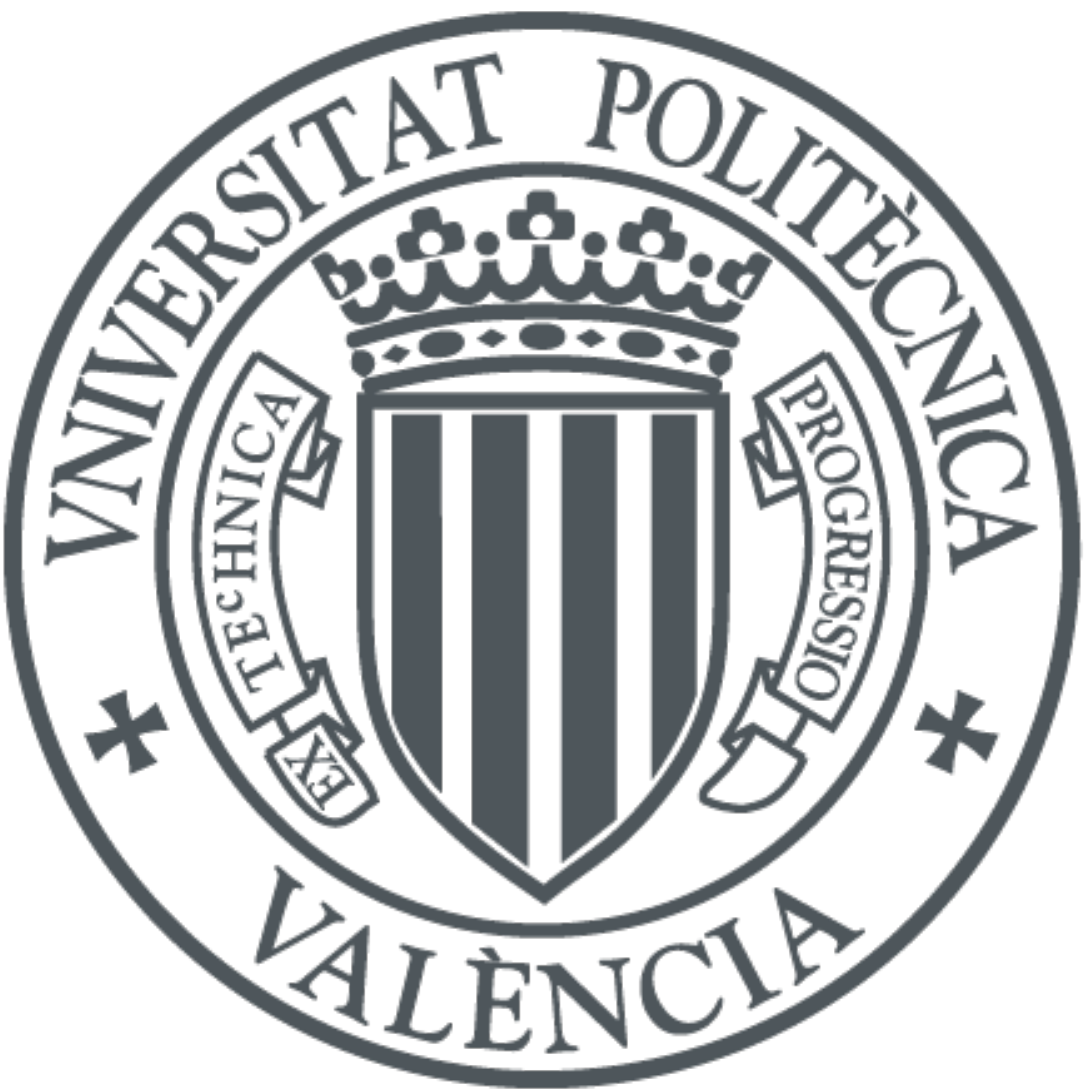

The final publication is available at

https://doi.org/10.1016/j.cor.2017.01.003

Copyright Elsevier

Additional Information 


\title{
The Multi-objective Assembly Line Worker Integration and Balancing Problem of Type-2
}

\author{
Mayron César O. Moreira \\ Departamento de Ciência da Computação - Universidade Federal de Lavras \\ Lavras, Brazil \\ mayron.moreira@dcc.ufla.br \\ Rafael Pastor \\ Institut d'Organització i Control de Sist. Indust. - Universitat Politècnica de Catalunya \\ Barcelona - Spain \\ rafael.pastor@upc.edu \\ Alysson M. Costa \\ School of Mathematics and Statistics - University of Melbourne \\ Melbourne - Australia \\ alysson.costa@unimelb.edu.au \\ Cristóbal Miralles \\ Departament d'Organització d'Empreses - Universitat Politècnica de València \\ Valencia - Spain \\ cmiralles@omp.upv.es
}

\begin{abstract}
The consideration of worker heterogeneity in assembly lines has received a fair amount of attention in the literature in the past decade. Most of this exploration uses as motivation the example of assembly lines in sheltered work centers for the disabled. Only recently has the community started looking at the situation faced in assembly lines in the general industrial park, when in the presence of worker heterogeneity. This step raises a number of questions around the best way to incorporate heterogeneous workers in the line, maximizing their integration while maintaining productivity levels. In this paper we propose the use of Miltenburg's regularity criterion and cycle time as metrics for integration of workers and productivity, respectively. We then define, model and develop heuristics for a line balancing problem with
\end{abstract}


these two goals. Results obtained through an extensive set of computational experiments indicate that a good planning can obtain trade-off solutions that perform well in both objectives.

Keywords: Assembly Line Balancing, disabled workers, worker integration, regularity criterion.

\section{Introduction}

Traditional assembly line balancing (ALB) research focuses on the simple assembly line balancing problem (SALBP) initially defined by Baybars (1986) through several well-known simplifying hypotheses. This classical singlemodel problem consists of finding the best feasible assignment of tasks to stations so that precedence constraints are fulfilled. Two basic versions of this problem are called type-1, in which the cycle time, $c$, is given, and the aim is to minimize the number of needed workstations; and type-2, used when there is a given number of workstations, $m$, and the goal is to minimize the cycle time (Scholl, 1999).

Research focus has later changed in order to consider features present in more realistic industrial settings (Scholl and Becker, 2006; Becker and Scholl, 2006; Boysen et al., 2007, 2008; Battaïa and Dolgui, 2013) such as different line layouts, specific assignment constraints and multi-model lines, to cite a few. An important subset of this research has been interested in generalizing one of the main assumptions of the SALBP that states that workers have equal task processing times. In this context, some authors have considered the workforce with different levels of performance (Mansoor, 1968; Bartholdi and Eisenstein, 1996; Gel et al., 2002; Corominas et al., 2008; Koltai et al., 2014, e.g.). We are particularly interested in another variant, in which heterogeneity is more pronounced, configuring the so called assembly line worker assignment and balancing problem (ALWABP) (Miralles et al., 2007). In this problem, inspired by assembly lines in sheltered work centers for the disabled (SWDs), workers are highly heterogeneous. Indeed, not only each worker might have a specific processing time for each task, but also each worker has a set of tasks that they can not execute, called incompatible tasks.

Research on the ALWABP has concentrated on the obtention of efficient exact (Miralles et al., 2008; Borba and Ritt, 2014; Vilà and Pereira, 2014) and heuristic methods (Moreira and Costa, 2009; Blum and Miralles, 2011; 
Moreira et al., 2012; Mutlu et al., 2013; Borba and Ritt, 2014; Vilà and Pereira, 2014; Polat et al., 2015; Bouajaja and Dridi, 2015) to solve this double assignment problem in which tasks and workers must be simultaneously matched to workstations. Concurrently, as in the rest of the ALB literature, different line features have been studied in the context of the ALWABP (Araújo et al., 2012, 2015; Cortez and Costa, 2015). All these studies have kept the original motivation of sheltered work centers and therefore considers a full set of heterogeneous workers.

In spite of the extreme social importance of SWDs and its significant contribution in providing job opportunities for persons with disabilities, it stills configure a somehow segregating space, not completely achieving the ultimate goal of providing a fully societal integration. This question has recently started to be addressed: Moreira et al. (2015b) extended some ALWABP models and algorithms to conventional companies, defining the assembly line worker integration and balancing problem (ALWIBP). In this study, the authors consider the balancing of lines in which only a percentage of workers have heterogeneous capabilities, mimicking the situation of assembly lines in conventional industrial settings that open job opportunities for persons with disabilities. Moreira et al. (2015a) have also considered the robustness of the obtained solutions when faced with task time variability.

These two articles have opened a new avenue for research, in which particularities associated with the operation of mixed (with conventional and heterogeneous workers) lines must be addressed. One new practical aspect of the problem is the need to evenly distribute workers with special characteristics along the lines. This becomes an important feature in conventional industries mixed (as defined above) lines, in which one usually aims to evenly distribute workers with disabilities. This generate configurations that do not segregate workers with disabilities in clusters and allow them to fully cooperate with more experienced workers. Interestingly, the even distribution of a subset of workers along the line may also be an important ingredient in some SWDs, in which a few number of more experienced workers - or monitors - might also be present and one would like to have them evenly spaced throughout the line.

In this article, we address the research question of proposing models and solution methods to provide balancing solutions that combine these two objectives, integration and productivity. Productivity is considered by the minimization of cycle time, as it is usually done in the literature. We provide a metric for even worker distribution based on Miltenburg (1989) production 
variation rate criteria, which is adapted and linearized in a mixed-integer program (Section 3). We also develop fast heuristic methods in order to obtain good quality solutions for larger problems in short computational times (Section 4). An extensive set of experiments validate the efficiency of the methods and allow for a analysis of the existing trade-off between these two possibly conflicting objectives (Section 5). A number of conclusions and avenues for future research end this paper in Section 6 .

\section{Formal definitions and a mathematical model for the ALWIBP-2}

Let $S=\{1, \ldots, m\}$ be a set of ordered workstations, $W=\{1, \ldots, d\}$ be a set of heterogeneous workers and $N=\{1, \ldots, n\}$ be a partially ordered set of tasks. Consider that there are precedence constraints among tasks such that for $i, j \in N, i \lessdot j$ indicates that task $i$ is an immediate predecessor of task $j$. The goal of the ALWIBP-2 is, given a fixed number of workstations, $m$, to find an assignment of tasks and workers minimizing the cycle time, $c$, such that precedence relationships and incompatibilities are respected.

To model this problem, define $F_{i}=\{j \in N \mid i \lessdot j\}$ as a set of immediate successors of task $i \in N$ and $t_{i}\left(t_{w i}\right)$ as the execution time of task $i$ when assigned to a conventional (heterogeneous) worker. We assume that $t_{i}$ is the reference value for the execution time of task $i$ and $t_{i} \leq t_{w i}$, for each $w \in W$. Let $W_{i} \subseteq W$ be the subset of heterogeneous workers that are able to execute task $i \in N$ and $N_{w} \subseteq N$ be the set of tasks that worker $w \in W$ is able to perform, $N_{w}=\left\{i \in N \mid w \in W_{i}\right\}$. Consider $x_{s i}$ as binary variables equal to 1 only if task $i \in N$ is assigned to workstation $s \in S$ and $y_{s w}$ as binary variables equal to 1 only if worker $w \in W$ is assigned to workstation $s \in S$. Thus, taking $l_{w}, w \in W$, as positive constants, a mixed integer model for the ALWIBP-2 can be written as:

M1: minimize $c$

subject to

$$
\begin{array}{ll}
\sum_{s \in S} x_{s i}=1 & i \in N \\
\sum_{i \in N} x_{s i} \geq 1 & s \in S \\
\sum_{s \in S} y_{s w}=1 & w \in W
\end{array}
$$




$$
\begin{array}{cl}
\sum_{w \in W} y_{s w} \leq 1 & s \in S \\
\sum_{\substack{s \in S ; \\
s \geq k}} x_{s i} \leq \sum_{\substack{s \in S ; \\
s \geq k}} x_{s j} & i \in N, j \in F_{i}, k \in S \backslash\{1\} \\
\sum_{i \in N} t_{i} x_{s i} \leq c & s \in S \\
\sum_{i \in N_{w}} t_{w i} x_{s i} \leq c+l_{w}\left(1-y_{s w}\right) & s \in S, w \in W \\
y_{s w} \leq 1-x_{s i} & s \in S, i \in N, w \notin W_{i} \\
x_{s i} \in\{0,1\}, & s \in S, i \in N \\
y_{s w} \in\{0,1\} & s \in S, w \in W \\
c \geq 0 . &
\end{array}
$$

The objective function (1) minimizes the cycle time of the assembly line. Constraints (2)-(3) guarantee that each task is assigned to a single workstation and that all workstations have at least one task, respectively. Constraints (4) state that each heterogeneous worker is assigned to a workstation, while Constraints (5) establish that a workstation can receive at most one worker. Precedence relations among tasks are enforced by Constraints (6). Constraints (7) and (8) ensure that the cycle time is respected in workstations without or with heterogeneous workers, respectively. Note that constants $l_{w}$ defined earlier must be sufficiently large to deactivate these latter constraints if $y_{s w}=0$. We use $l_{w}=\sum_{i \in N_{w}} t_{w i}-t_{i}$ which assumes the maximum additional time that a heterogeneous worker $w$ spends at a station in comparison to a "conventional" worker. Constraints (9) imply that tasks are not assigned to heterogeneous workers who are not able to execute them.

\section{Incorporating Miltenburg's criteria}

In this paper, we consider the ALWIBP-2 which minimizes the cycle time while trying to obtain a more appropriate assignment of workers. This second goal aims at distributing as evenly as possible the heterogeneous workers along the line, so that: $(i)$ "conventional" workers can assist them in the execution of their tasks (in SWDs, for example) or (ii) clusters of heterogeneous workers are avoided. In both cases, the main idea is to obtain a line balancing in which workers from a special set (monitors, in the case of lines in SWDs 
or heterogeneous workers, in the case of mixed lines) are evenly distributed along the line .

In order to evaluate this criterion, we adapt one of the regularity criteria proposed by Miltenburg (1989). The author introduces the product rate variation problem (known in the literature as PRV), which appears in justin-time production systems (JIT), especially in mixed-model assembly lines. The goal of a regular distribution of products is relevant in these environments since they aim at producing only necessary quantities and then, it is important to maintain the rate of usage of the line.

In mathematical terms, let $p$ be the quantity of different products and $\tilde{u}$ be the total number of units to sequence. Also, consider $u_{i}$ as the number of units of each product and $\beta_{i j}$ as the quantity of product $i$ sequenced until position $j$. Then, we can measure the regularity criterion of a schedule, $r$, by:

$$
r=\sum_{i=1}^{p} \sum_{j=1}^{\tilde{u}}\left(\beta_{i j}-\frac{u_{i}}{\tilde{u}} j\right)^{2}
$$

Kubiak and Sethi (1991) proposed an assignment formulation for the PRV which can be extended for more general objective functions. Later, Bautista et al. (1996a) considered relations between the PRV and the apportionment problem in order to state some useful properties to solve the former one. More studies concerning other JIT scheduling problems as well as different sequencing metrics can be found in (Monden, 1983; Miltenburg and Sinnamon, 1989; Kubiak, 1993; Bautista et al., 1996b; Duplaga and Bragg, 1998; Lebacque et al., 2007; Boysen et al., 2009).

In our context and considering the case of a mixed line, let $v_{s}\left(z_{s}\right)$ be the number of "conventional" (heterogeneous) workers assigned in stations up to (and including) station $s$. Then, $r$ is computed as follows:

$$
r=\sum_{s \in S}\left[\left(z_{s}-\frac{|W|}{|S|} s\right)^{2}+\left(v_{s}-\frac{|S|-|W|}{|S|} s\right)^{2}\right]
$$

Note that we do not need to consider both terms in (14), since the regularity of the distribution of workers with disabilities implies a proper placement of the others. For this purpose, see that $v_{s}=s-z_{s}, s \in S$. Therefore equation (14) can be rewritten as: 


$$
\begin{array}{r}
\mathrm{r}=\sum_{s \in S}\left[\left(z_{s}-\frac{|W|}{|S|} s\right)^{2}+\left(s-z_{s}-\frac{|S|-|W|}{|S|} s\right)^{2}\right]= \\
\sum_{s \in S}\left[\left(z_{s}-\frac{|W|}{|S|} s\right)^{2}+\left(-z_{s}+\frac{|W|}{|S|} s\right)^{2}\right]= \\
\sum_{s \in S}\left[2\left(z_{s}-\frac{|W|}{|S|} s\right)^{2}\right]=2 \sum_{s \in S}\left[\left(z_{s}-\frac{|W|}{|S|} s\right)^{2}\right] .
\end{array}
$$

We now consider $\pi_{s}$ as binary variables equal to 1 if a disabled worker is assigned to workstation $s \in S$. Model $M 2$ designed for the worker regularity problem can be written as follows:

$$
\text { M2: minimize } \sum_{s \in S}\left(z_{s}-\frac{|W|}{|S|} s\right)^{2}
$$

subject to

$$
\begin{aligned}
\sum_{s \in S} \pi_{s}=|W| & \\
z_{s}=\sum_{\substack{s^{\prime} \in S ; \\
s^{\prime} \leq s}} \pi_{s^{\prime}} & \forall s \in S \\
\pi_{s} \in\{0,1\} & \forall s \in S \\
z_{s} \in \mathbb{Z} & \forall s \in S .
\end{aligned}
$$

The objective function (16) prioritizes the uniform distribution of workers. Constraints (17) guarantee the assignment of all heterogeneous workers in the assembly line, while constraints (18) compute the cumulative amount of them for each position of the line. Constraints (19) and (20) are integrality constraints.

The resulting model is clearly nonlinear. In order to linearize it, let $h_{(s)}$ and $\kappa_{(s)}$ be the minimum and maximum possible number of heterogeneous workers assigned until station $s, h_{(s)}=\max \{0,|W|-|S|+s\}$ and $\kappa_{(s)}=$ $\min \{s,|W|\}$, respectively, and $K_{s}=\left\{h_{(s)}, \ldots, \kappa_{(s)}\right\}$ be a set of possible values for the number of these workers assigned in station $s$ or prior to it. Moreover, 
let $\gamma_{s \kappa}$ be binary variables equal to 1 only if there are $\kappa$ heterogeneous workers allocated until workstation $s$. A linearized version of model M2 follows:

$$
\text { M3: minimize } \sum_{s \in S} \sum_{\kappa \in K_{s}}\left[\kappa^{2}-2 \kappa\left(\frac{|W|}{|S|} s\right)+\left(\frac{|W|}{|S|} s\right)^{2}\right] \gamma_{s \kappa}
$$

subject to

$$
\begin{gathered}
\sum_{\kappa \in K_{s}}^{(17),(19) \text { and }} \gamma_{s \kappa}=1 \quad s \in S \\
\sum_{\substack{s^{\prime} \in S ; \\
s^{\prime} \leq s}} \pi_{s^{\prime}}=\sum_{\kappa \in K_{s}} \kappa \gamma_{s \kappa} \quad s \in S \\
\gamma_{s \kappa} \in\{0,1\} \quad s \in S, \kappa \in K_{s} .
\end{gathered}
$$

The objective function (21) is a linearized version of the sum of the squared deviations of ideal and real number of heterogeneous workers and "conventional" ones. Constraints (22)-(23) compute the number of heterogeneous workers partially distributed along the assembly line.

Observe that this model is more appropriate if we deal with assembly lines with more "conventional" workers than heterogeneous ones, since the cardinality of set $K_{s}, s \in S$, will be lower and hence, the model will be more compact. For assembly lines with a larger number of heterogeneous workers (and a few monitor workers, such in SWDs), we indicate a formulation using the second term of equation (14) as objective function in order to reduce the number of variables in the process of linearization, which is analogous to the previous one. We also point out that the methods and models are valid when there is the general objective of distributing a given subset of workers along the line.

Let $s^{p}=\left(\begin{array}{llll}s_{1}{ }^{p} & s_{2}{ }^{p} \ldots s_{m}^{p}\end{array}\right)$ be a $m$-tuple representing the positions of heterogeneous workers in the line. Parameter $s_{k}^{p}=1$ if there is a heterogeneous worker $w \in W$ in station $k \in S$.

Given a tuple $s^{p}$, we can obtain solutions by minimizing the cycle time and adding a set of constraints to ensure that heterogeneous workers are assigned to the indicated positions. This yields model $M 4$, which is presented below: 
subject to

$$
\sum_{w \in W} y_{s w}^{(2)-(12) \text { and }}-s_{s}^{p}=0 \quad \forall s \in S .
$$

Constraints (26) force heterogeneous workers to be assigned to a predetermined set of workstations, defined by the $m$-tuple $s^{p}$.

Let $\bar{p}$ be the number $m$-tuples $s^{p}$ to be generated, and $P_{\bar{p}}$ be the set of these configurations, where $p \in\{1, \ldots, \bar{p}\}$. We generate set $P_{\bar{p}}$ via a worker regularity generator model (WRGM), which extends model $M 3$ in order to avoid that the same configuration be found more than once:

$$
W R G M \text { : minimize } \sum_{s \in S} \sum_{\kappa \in K_{s}}\left[\kappa^{2}-2 \kappa\left(\frac{|W|}{|S|} s\right)+\left(\frac{|W|}{|S|} s\right)^{2}\right] \gamma_{s \kappa}
$$

subject to

(17), (19), (22)-(24) and

$$
\sum_{k \in S} s_{k}^{p^{\prime}} \pi_{k} \leq|W|-1 \quad p^{\prime} \in\{1, \ldots, p-1\}, s^{p^{\prime}} \in P_{p-1} .
$$

Constraints (28) prohibit that worker distribution $\pi$ is identical to the ones already in set $P_{\bar{p}}$. In order to evaluate the cycle time for the worker distributions, we call model $M_{4}$ for each $s^{p} \in P_{\bar{p}}, p=1, \ldots, \bar{p}$. Note that in the end of each execution, we will have the information of which worker is assigned to which workstations. Algorithm 1 summarizes the procedure.

In the Algorithm, during the loop in lines 3-5, we apply the auxiliary function solveM $4\left(s^{p}\right)$ that has as input a tuple $s^{p}$ and solves the $M_{4}$ model. In the end, the algorithm returns the best cycle time value for each tested distribution $s^{p}$. 
$\overline{\text { Algorithm } 1 \text { Evaluation of the cycle time for each worker regularity distri- }}$ bution

1: given $P_{\bar{p}}$ (obtained with model WRGM);

2: Consider $c^{\bar{p}}=\left\{\hat{c}^{1} \hat{c}^{2} \ldots \hat{c}^{\bar{p}}\right\}$ a $\bar{p}$-tuple that measures the cycle time for each configuration $p \in\{1, \ldots, \bar{p}\}$;

3: for all $p \in\{1, \ldots, \bar{p}\}$ do

4: $\quad c^{p} \leftarrow$ solveM4 $\left(s^{p}\right)$;

5: end for

6: $\operatorname{return} c^{\bar{p}}$.

\section{Heuristic methods}

In this section, we present three constructive heuristics for the ALWIBP2 with productivity and integration objectives. As in the previous section, all three procedures start by generating $P_{\bar{p}}$, the set of tuples containing the heterogeneous workers positions to be tested. The second step concerns the generation of task and worker assignments respecting these configurations. The second step differs in the three different heuristics, as explained in the following.

\subsection{Phase 1: Generation of the type worker configurations}

The size of set $P_{\bar{p}}$ grows exponentially with the number of workstations. For instance, if we take an assembly line with 30 workstations and 9 heterogeneous workers, we have $\left|P_{\bar{p}}\right|=C_{9}^{30}=14.307 .150$ possible configurations. Using model WRGM presented earlier, we consider the $\tilde{p}=30$ best solutions in terms of the adaptation of Miltenburg's criterion proposed earlier.

\subsection{Phase 2: Task and worker assignments}

Given set $P_{\bar{p}}$ obtained in Phase 1 , we perform the task and worker assignments according to three different procedures: a M4-based heuristic (MH, Section 4.2.1), a worker regularity constructive heuristic (WRCH, Section 4.2.2) and a worker regularity constructive heuristic with randomness (WRCHR, 4.2.3).

\subsection{1. $\mathrm{MH}:$ M4 model based heuristic}

The $M H$ performs the task and worker assignments using Algorithm 1 for $\bar{p}$ equal to $\tilde{p}$. For each configuration $s^{p} \in P_{\tilde{p}}, p \in\{1, \ldots, \tilde{p}\}$, the Algorithm is allowed to run for $\mathcal{T}_{p}$ seconds. If the model is not solved to optimality in this time, the best feasible solution is returned. 


\subsubsection{WRCH: worker regularity constructive heuristic}

The task assignment phase of the WRCH is based on the ALWABP-2 constructive heuristic $(\mathrm{CH})$ proposed by Moreira et al. (2012). The $\mathrm{CH}$ initially estimates a range of cycle times. For each cycle time value (in increasing order), the algorithm tries to assign tasks and workers to stations subject to precedence constraints. Assignment is made sequentially, in a workstationoriented fashion and makes use of worker and task priority rules (Moreira et al., 2012). The procedure stops when the solution found is feasible for the current cycle time. The authors consider 16 task priority rules and 3 worker allocation criteria.

We perform task and worker assignments according to configurations $s^{p} \in$ $P_{\tilde{p}}, p \in\{1, \ldots, \tilde{p}\}$. The worker-assignment procedure in $\mathrm{CH}$ is modified in order to only assign heterogeneous workers to the positions indicated in $s^{p}$.

\subsubsection{WRCHR: worker regularity constructive heuristic with randomness}

We extend the WRCH by including in task assignment. Let $\mathcal{N} \subseteq N$ be

the set of candidate tasks such that $i \in \mathcal{N}$ if and only if all predecessors of $i$ have already been assigned and the insertion of $i$ on the current station respects the cycle time constraints. Consider $\mathcal{R} \subseteq \mathcal{N}$ as the set of tasks with the best value of the priority rules adopted. The worker regularity constructive heuristic with randomness (WRCHR) adapts the task selection of $\mathrm{CH}$ randomly choosing with the same probability a task $j \in \mathcal{R}$ at each step. The task and worker priority rules remain the same as presented in the WRCH.

\section{Experimental study}

We have carried out an experimental study over a new benchmark proposed for the ALWIBP-2. In Section 5.1, we introduce this set of instances. Section 5.2 presents the numerical tests concerning the ALWIBP-2 model (Section 5.2.1) and heuristics (Section 5.2.2).

\subsection{Benchmark scheme}

The proposed benchmark for the ALWIBP-2 considers conventional assembly lines with a parcel of heterogeneous workers. We select the 100 instances from (Otto et al., 2013) for each family of instances with 50 (middlesized) and 100 (large-sized) tasks. These examples were the same used in (Moreira et al., 2015b), and have the following characteristics: (i) mixed 
precedence graphs (with chain and bottleneck structures); (ii) "high" and "low" order strengths; and (iii) task times generated according to "peak at the bottom" and "bimodal" distributions.

Precedence graphs are kept as in the original instances, as well as task execution times $t_{i}$. In order to generate task execution times for heterogeneous workers, we use uniform distributions that depend on the kind of instance being generated (low or high task variability and low or high task $\times$ worker incompatibilities). The parameters used are as follows:

- Variability of task execution times (Var): "low" $\left(U\left[t_{i}, 2 t_{i}\right]\right)$ and "high" $\left(U\left[t_{i}, 5 t_{i}\right]\right)$

- Task/worker incompatibilities (Inc): "low" (10\% of total of tasks) and "high" (20\% of total of tasks);

Taking both factors, we have a total of 4 instances generated for each original instance from (Otto et al., 2013). The number of stations $m$ considered for each of them is the same as its corresponding SALBP instance. The quantity of "conventional" and heterogeneous workers is defined by $m-\lceil\mu \times m\rceil$ and $\lceil\mu \times m\rceil$, where $\mu$ is a parameter indicating the estimated percentage of heterogeneous workers in the assembly line. We use three values for $\mu$, given by $10 \%, 20 \%$ and $30 \%$, generating a total of 1200 instances for each group of 50 and 100 tasks.

\subsection{Computational study}

In the following sections, we measure the performance of both models and algorithms proposed for the ALWIBP-2 and its variant with worker regularity goal. The methods were coded in $\mathrm{C}++$, in the Linux operational system. Computational tests were conducted in a $\operatorname{Intel}(\mathrm{R}) \mathrm{Xeon}(\mathrm{R}) \mathrm{X} 5675$ 3.07GHz 96 GB RAM machine, using IBM CPLEX 12.6 with 1 thread and $6 \mathrm{~GB}$ as the size limit of the search tree.

\subsubsection{Experiment 1: M1 model}

We set up the solution time limit for model $M 1$ as 3,600s. Tables 1 and 2 use the following criteria as average performance measures:

- $\Delta_{c}(\%)$ : percentage of proved optimal solutions;

- $\Upsilon_{1}^{*}(\%)$ : percentage increase in the ALWIBP-2 cycle time when compared to the reference solution for the SALBP-2; 
- $\operatorname{Gap}(\%)$ : optimality gap obtained by CPLEX in the allowed time limit;

- $\mathrm{t}(\mathrm{s})$ : run time.

In Table 1, the results show that the model proved optimal solutions in approximately $69 \%$ of instances. Note that the reduced gaps indicate the possibility to obtain a larger number of optimal solutions, even though gaps of optimality have not been closed. Incorporating heterogeneous workers and regularity distribution criteria had an effect of degrading the cycle time, but in our tests this was kept in reasonable values (an average $6.5 \%$ increase in cycle time). This seems like a reasonable price to pay, specially considering that the generated instances include more heterogeneous workers (a minimum of $10 \%$, in the easiest instances) than it is usually required by most national legislations.

Table 2 shows the results obtained for instances with 100 tasks. As expected, the number of optimal solutions proved and the average gap of optimality have worsened due to the higher complexity of these examples. However, the greater flexibility to assign tasks to workstations in this context reduced the increased percentage of cycle time compared with SALBP-2 large-sized solutions. Finally, the computational time to solve both groups of instances proved to be reasonable and applicable in operational planning in ALWIBP-2 environments.

\subsubsection{Experiment 2: ALWIBP-2 approaches}

This section addresses comparisons among the three heuristics applied to the ALWIBP-2 with worker regularity criterion. Preliminary tests have been conducted to establish the parameters of the algorithms. According to our experiments, we take $\tilde{p}$ and $\mathcal{T}_{p}, p \in\{1, \ldots, \tilde{p}\}$, equal to 30 solutions and 1,800 s, respectively. Furthermore, the WRCHR was run 20 times in order to deal with its stochastic characteristic. Still considering this algorithm, the maximum quantity of candidate tasks (i.e. the set $\mathcal{R}$ ) selected in the assignment phase was fine-tuned to 5 . The columns of Table 3 present the evaluation criteria:

- $\delta_{c}(\%)$ : average percentage of proved optimal solutions;

- $\Upsilon_{2}^{*}(\%)$ : average gap of the best cycle time found in all type worker configurations compared with SALBP-2 reference solution; 
Table 1: ALWIBP-2 model - results of middle instances (50 tasks).

\begin{tabular}{rccrrrr}
\hline$\mu$ & Var & Inc & $\Delta_{c}(\%)$ & $\Upsilon_{1}^{*}(\%)$ & $\operatorname{Gap}(\%)$ & $\mathrm{t}(\mathrm{s})$ \\
\hline 10 & 2 & 10 & 70 & 2.2 & 0.3 & 1,168 \\
& & 20 & 68 & 2.6 & 0.3 & 1,277 \\
& 5 & 10 & 72 & 5.3 & 0.2 & 1,102 \\
& & 20 & 75 & 5.7 & 0.2 & 1,087 \\
& & & & & & \\
20 & 2 & 10 & 61 & 4.0 & 0.5 & 1,487 \\
& & 20 & 67 & 4.6 & 0.4 & 1,264 \\
& 5 & 10 & 73 & 10.0 & 0.3 & 1,067 \\
& & 20 & 73 & 10.7 & 0.3 & 1,068 \\
30 & 2 & 10 & 66 & 6.3 & 0.7 & 1,335 \\
& & 20 & 61 & 6.6 & 0.7 & 1,482 \\
& 5 & 10 & 74 & 15.2 & 0.6 & 1,116 \\
& & 20 & 70 & 16.6 & 0.8 & 1,175 \\
& & & & & & \\
& Avg. & & & 7.5 & 0.4 & 1,219 \\
\hline
\end{tabular}


Table 2: ALWIBP-2 model - results of large instances (100 tasks).

\begin{tabular}{rccrrrr}
\hline$\mu$ & Var & Inc & $\Delta_{c}(\%)$ & $\Upsilon_{1}^{*}(\%)$ & $\operatorname{Gap}(\%)$ & $\mathrm{t}(\mathrm{s})$ \\
\hline 10 & 2 & 10 & 32 & 1.6 & 1.2 & 2,511 \\
& & 20 & 34 & 1.8 & 1.3 & 2,492 \\
& 5 & 10 & 26 & 3.8 & 2.1 & 2,693 \\
& & 20 & 28 & 4.0 & 2.1 & 2,623 \\
& & & & & & \\
20 & 2 & 10 & 21 & 3.0 & 2.3 & 2,945 \\
& & 20 & 26 & 3.2 & 2.4 & 2,771 \\
& 5 & 10 & 22 & 6.7 & 4.2 & 2,884 \\
& & 20 & 23 & 7.2 & 4.5 & 2,796 \\
30 & 2 & 10 & 3 & 5.0 & 4.4 & 3,388 \\
& & 20 & 6 & 5.4 & 4.6 & 3,358 \\
& 5 & 10 & 2 & 11.4 & 9.0 & 3,491 \\
& & 20 & 2 & 12.3 & 9.6 & 3,466 \\
& & & & & & \\
& Avg. & & & 5.5 & 4.0 & 2,951 \\
\hline
\end{tabular}


- $\Upsilon_{2}^{* \rho}(\%)$ : average gap of the best cycle time found in $\rho$ best type worker configurations compared with SALBP-2 reference solution;

- $\bar{\Upsilon}_{2}^{\rho}(\%)$ : average gap of the cycle times found in $\rho$ best type worker configurations compared with SALBP-2 reference solution;

- $\bar{\Upsilon}_{2}(\%)$ : average gap of the cycle times found in all type worker configurations compared with SALBP-2 reference solution;

In our experiments, we adopt $\rho$ equal to $20 \%$ of the quantity of the best type worker distribution (without repetition). In the metrics, we consider worker placements that all the three heuristic procedures found at least feasible solutions. According to the Column $\Upsilon_{2}^{*}$ of Table 3, we pay a price of approximately $8 \%$ of increase cycle time when we also prioritize the regular distribution of workers along the line, comparing with a productive rate of an ordinary system. Taking $\rho$ best type worker configurations concerning regularity, note that these figures remain close to the best solutions. Analyzing the overall average (Column $\bar{\Upsilon}_{2}$ ), we see that the MH performed well in all scenarios tested. The results also show that the algorithms WRCH and WRCHR obtained solutions close to the ones obtained by the MH. It is important to highlight that the diversified solutions obtained by the WRCHR as consequence of its random task choice were profitable considering the criteria evaluated. As depicted in Column " $\delta_{c}$ ", the ALWIBP-2 model were solved to optimality for most of type worker placements.

Table 4 corroborates the good performance of the three algorithms implemented. In Column " $\delta_{c}$ ", due to the complexity to solve problems of this magnitude, the number of optimal solutions found by the ALWIBP-2 model reduced. As we observed in the previous section, cycle time values improve when we deal with large-sized instances. Note that average represented by Column $\Upsilon_{2}^{*}$ of MH is even lower than the one found by the ALWIBP-2 model (Column $\Upsilon_{1}^{*}$ of Table 2), due to the model inability to find better feasible solutions in the allowed time limit.

We also investigate the impact of the priority rules proposed by Moreira et al. (2012) behind WRCH and WRCHR. Considering the first algorithm, we observed that the criteria based on task execution times obtained the best results. On the other hand, the stochastic features present in the task assignment phase of the WRCHR resulted in rules with similar behavior in both set of instances. 


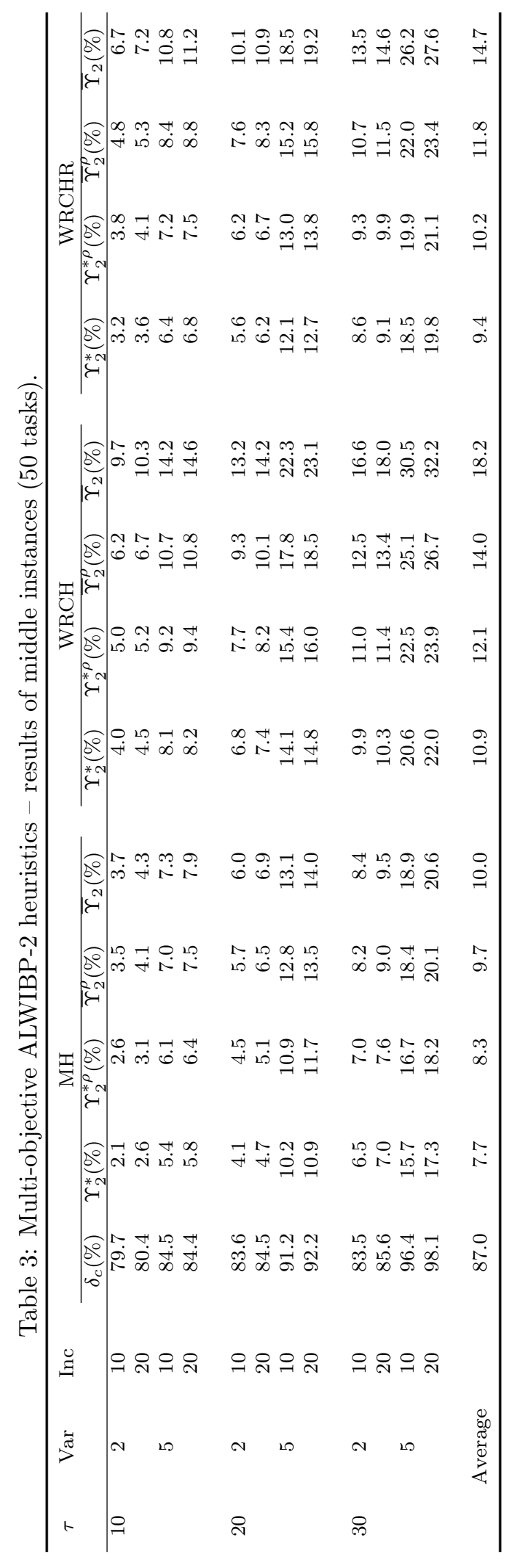




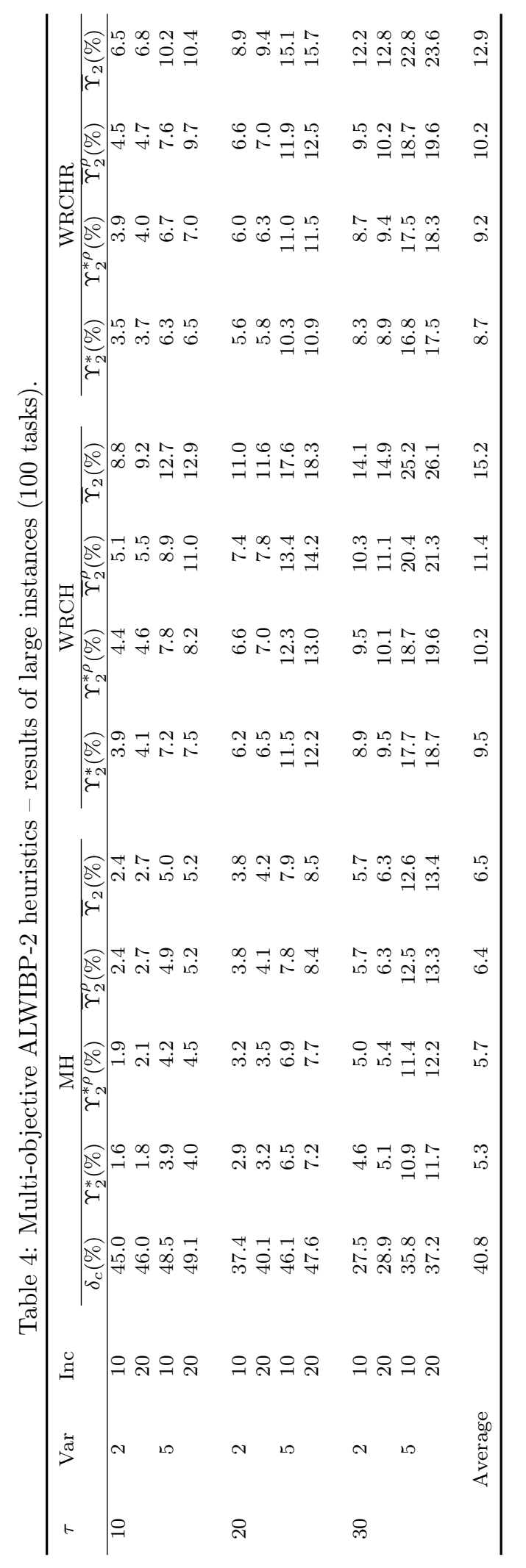


The idea of pre-determined stations to receive "conventional" workers and disabled ones can generate infeasible solutions, since there may not be task assignments that respect cycle time constraints or even task/worker feasibilities. Taking the middle-sized instances, we observed that the three heuristics have not found solutions in less than $0.8 \%$ of the configurations tested. For problems with 100 tasks, the MH obtained feasible solutions for all worker placements, while WRCH and WRCHR have not succeeded in $0.8 \%$, on average. The average computational time (in seconds) spent by the $\mathrm{MH}$, WRCH and WRCHR for solving the ALWIBP-2, for each configuration, was 354.00, 0.02, 0.20 seconds (middle-sized instances), and 1,170.52, 0.09, 1.64 seconds (large-sized instances), respectively.

Cycle time and Miltenburg's regularity criterion can be conflicting objective functions. We illustrate the interaction between them comparing the three methods through a Pareto dominance concept. For this purpose, we choose two representative instances which have solutions with the larger variety of both criteria. Therefore, let $f_{1}, \ldots, f_{\text {obj }}$ be functions to be minimized. A solution $x$ dominates $y$ if $f_{i}(x) \leq f_{i}(y), i=1, \ldots, o b j$ and $f_{i}(x)<f_{i}(y)$, for at least one $i$. We say that $x$ is Pareto optimal if there is no $y$ such that $y$ dominates $x$.

In Figure 1, we present a graphic for a typical middle (Figure 1(a)) and a typical large instance (Figure $1(\mathrm{~b})$ ) with $\mu=10 \%$. The superiority of $\mathrm{MH}$ is confirmed in both cases against WRCH and WRCHR algorithms. The dominant set is almost integrally composed by the solutions from the model-based method. Nevertheless, the heuristic methods that do not require the solution of a mixed-integer program are still valuable given their computational efficiency and their scalability to even larger lines. 


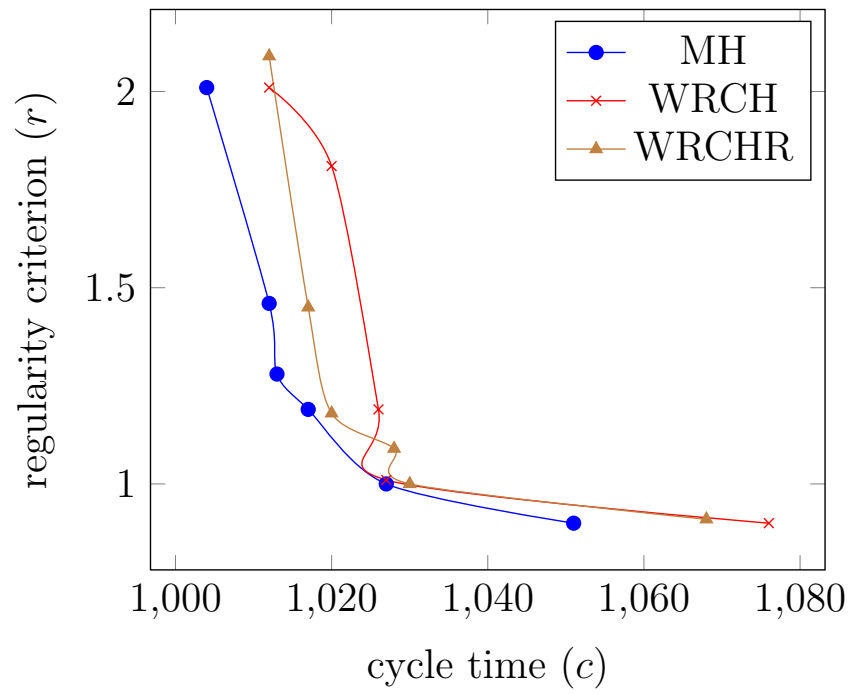

(a) $\mathrm{MH}, \mathrm{WRCH}$ and WRCHR: dominant sets for a middle instance with $\mu=10 \%$.

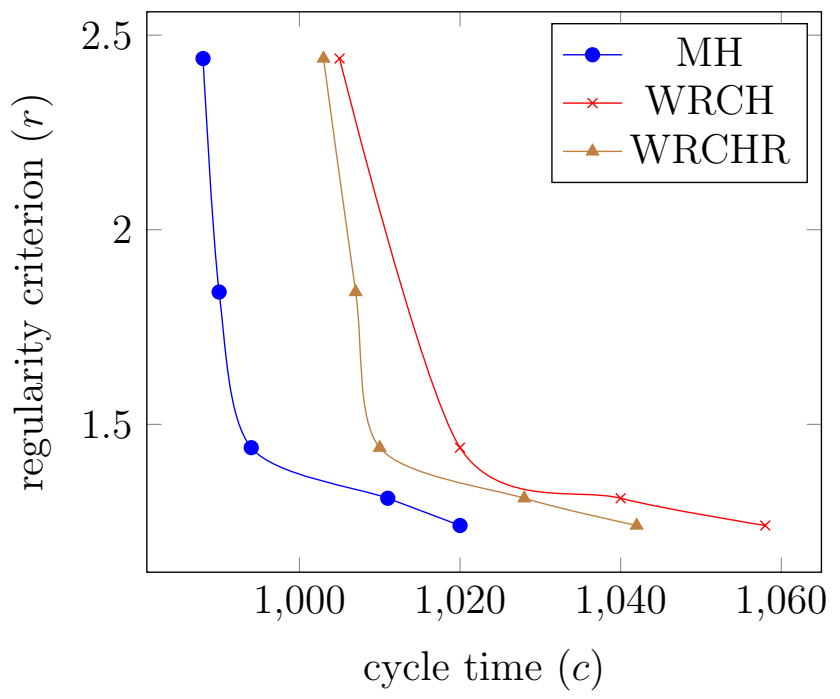

(b) $\mathrm{MH}, \mathrm{WRCH}$ and WRCHR: dominant sets for a large instance with $\mu=10 \%$.

Figure 1: Dominant sets of two instances for MH, WRCH and WRCHR algorithms.

\section{Conclusions}

In this paper we model and propose algorithms to solve an assembly line balancing problem where one aims to integrate workers with very different 
task execution times in the line. This problem is motivated by the situation faced in sheltered work centers for the disabled and in conventional assembly lines that employ a number of persons with disabilities.

We adapt Miltenburg's regularity criteria in order to obtain even distribution of a special set of workers (workers with disabilities or monitor workers) along the line, in order to avoid clustering of workers and promote a higher level of integration. A second objective is to maintain productivity levels.

Our algorithms present two stages. In the first stage, possible worker distributions are obtained and, in the second stage, they are used to obtain the worker and task assignments. Our results indicate that not only heterogeneous workers can be integrated in the line without major productivity losses, but also that the regularity distribution goal can be easily incorporated. This evidence becomes very important to encourage those companies that, within their CSR policies or pushed by emerging legal issues, are eager to integrate disabled people in their workforce.

Future research lines include the exploration of different assembly line aspects such as different layouts and special precedence constraints, which can be important in practical settings. There is also the need to investigate the practical efficiency of the integration metric used.

\section{Acknowledgements}

This research was supported by CAPES-Brazil and MEC-Spain (coordinated project CAPES DGU 258-12/PHB2011-0012-PC) and by FAPESPBrazil.

\section{References}

Araújo, F. F. B., Costa, A. M., Miralles, C., 2012. Two extensions for the assembly line worker assignment and balancing problem: parallel stations and collaborative approach. International Journal of Production Economics 140, 483-495.

Araújo, F. F. B., Costa, A. M., Miralles, C., 2015. Balancing parallel assembly lines with disabled workers. European Journal of Industrial Engineering 9, 344-365.

Bartholdi, J. J., Eisenstein, D. D., 1996. A production line that balances itself. Operations Research 44, 21-34. 
Battaïa, O., Dolgui, A., 2013. A taxonomy of line balancing problems and their solution approaches. International Journal of Production Economics $142,259-277$.

Bautista, J., Companys, R., Corominas, A., 1996a. A note on the relation between the product rate variation (PRV) problem and apportionment problem. Journal of the Operational Research Society 47, 1410-1414.

Bautista, J., Companys, R., Corominas, A., 1996b. Heuristics and exact algorithms for solving Monden problem. European Journal of Operational Research 88, 101-113.

Baybars, I., 1986. A survey of exact algorithms for the simple assembly line balancing problem. Management Science 32, 909-932.

Becker, C., Scholl, A., 2006. A survey on problems and methods in generalized assembly line balancing. European Journal of Operational Research 168, 694-715.

Blum, C., Miralles, C., 2011. On solving the assembly line worker assignment and balancing problem via beam search. Computers \& Operations Research 38, 328-339.

Borba, L., Ritt, M., 2014. A heuristic and a branch-and-bound algorithm for the assembly line worker assignment and balancing problem. Computers \& Operations Research 45, 87-96.

Bouajaja, S., Dridi, N., Nov 2015. Research on the optimal parameters of aco algorithm for a human resource allocation problem. In: Service Operations And Logistics, And Informatics (SOLI), 2015 IEEE International Conference on. pp. 60-65.

Boysen, N., Fliedner, M., Scholl, A., 2007. A classification of assembly line balancing problems. European Journal of Operational Research 183, 674693.

Boysen, N., Fliedner, M., Scholl, A., 2008. Assembly line balancing: which model to use when? International Journal of Production Economics 111, 509-528. 
Boysen, N., Fliedner, M., Scholl, A., 2009. Sequencing mixed-model assembly lines: Survey, classification and model critique. European Journal of Operational Research 192, 349-373.

Corominas, A., Pastor, R., Plans, J., 2008. Balancing assembly line with skilled and unskilled workers. Omega 36, 1126-1132.

Cortez, P. M. C., Costa, A. M., 2015. Sequencing mixed-model assembly lines operating with a heterogeneous workforce. International Journal of Production Research 53, 3419-3432.

Duplaga, E., Bragg, D., 1998. Mixed-model assembly line sequencing heuristics for smoothing component parts usage: a comparative analysis. International Journal of Production Research 36, 2209-2224.

Gel, E. S., Hopp, W. J., Van Oyen, M. P., 2002. Factors affecting opportunity of worksharing as a dynamic line balancing mechanism. IIE Transactions $34,847-863$.

Koltai, T., Tatay, V., Kalló, N., 2014. Application of the results of simple assembly line balancing models in practice: the case of a bicycle manufacturer. International Journal of Computer Integrated Manufacturing 27, 887-898.

Kubiak, W., 1993. Minimizing variations of production rates in just-in-time systems: a survey. European Journal of Operational Research 66, 259-271.

Kubiak, W., Sethi, S., 1991. A note on level schedules for mixed-model assembly lines in just-in-time production systems. Management Science 37, $121-122$.

Lebacque, V., Jost, V., Brauner, N., 2007. Simultaneous optimization of classical objectives in JIT sequencing. European Journal of Operational Research 182, 29-39.

Mansoor, E. M., 1968. Assembly line balancing: a heuristic algorithm for variable operator performance levels. Journal of Industrial Engineering 19, 618-629.

Miltenburg, J., 1989. Level schedules for mixed-model assembly lines in Justin-Time production systems. Management Science 35, 192-207. 
Miltenburg, J., Sinnamon, G., 1989. Scheduling mixed-model multi-level justin-time production systems. International Journal of Production Research 27, 1487-1509.

Miralles, C., García-Sabater, J. P., Andrés, C., Cardos, M., 2007. Advantages of assembly lines in sheltered workcentres for disabled. A case study. International Journal of Production Economics 110, 187-197.

Miralles, C., García-Sabater, J. P., Andrés, C., Cardos, M., 2008. Branch and bound procedures for solving the assembly line worker assignment and balancing problem: application to sheltered work centres for disabled. Discrete Applied Mathematics 156, 352-367.

Monden, Y., 1983. Toyota production system: practical approach to production management, 1st Edition. Industrial Engineering and Management Press, Boca Ratón.

Moreira, M. C. O., Cordeau, J.-F., Costa, A. M., Laporte, G., 2015a. Robust assembly line balancing with heterogeneous workers. Computers \& Industrial Engineering (In press).

Moreira, M. C. O., Costa, A. M., 2009. A minimalist yet efficient tabu search algorithm for balancing assembly lines with disabled workers. In: Anais do XLI SBPO. Porto Seguro, Brasil, pp. 660-671.

Moreira, M. C. O., Miralles, C., Costa, A. M., 2015b. Model and heuristics for the Assembly Line Worker Integration and Balancing Problem. Computers \& Operations Research 54, 64-73.

Moreira, M. C. O., Ritt, M., Costa, A. M., Chaves, A. A., 2012. Simple heuristics for the assembly line worker assignment and balancing problem. Journal of Heuristics 18, 505-524.

Mutlu, O., Polat, O., Supciller, A. A., 2013. An iterative genetic algorithm for the assembly line worker assignment and balancing problem of type-II. Computers \& Operations Research 40, 418-426.

Otto, A., Otto, C., Scholl, A., 2013. Systematic data generation and test design for solution algorithms on the example of salbpgen for assembly line balancing. European Journal of Operational Research 228, 33-45. 
Polat, O., Kalayci, C., Mutlu, O., Gupta, S., 2015. A two-phase variable neighbourhood search algorithm for assembly line worker assignment and balancing problem type-II: an industrial case study. International Journal of Production Research, 1-21.

Scholl, A., 1999. Balancing and sequencing of assembly lines. Physica-Verlag.

Scholl, A., Becker, C., 2006. State-of-the-art exact and heuristic solution procedures for simple assembly line balancing. European Journal of Operational Research 168, 666-693.

Vilà, M., Pereira, J., 2014. A branch-and-bound algorithm for assembly line worker assignment and balancing problems. Computers \& Operations Research 44, 105-114. 